\title{
Effects of less-invasive surfactant administration on oxygenation, pulmonary surfactant distribution, and lung compliance in spontaneously breathing preterm lambs
}

\author{
Hendrik J. Niemarkt ${ }^{1}$, Elke Kuypers ${ }^{1}$, Reint Jellema1 , Daan Ophelders ${ }^{1}$, Matthias Hütten ${ }^{1,2}$, Maria Nikiforou ${ }^{1}$, Angela Kribs ${ }^{3}$ and \\ Boris W. Kramer ${ }^{1}$
}

\begin{abstract}
BACKGROUND: A new technique was proposed to administer surfactant to spontaneous breathing preterm infants by placing a thin catheter through the vocal cords. This technique was not studied with respect to oxygenation, gas exchange, surfactant distribution, and lung mechanics. We tested the technique of less-invasive surfactant administration (LISA) in a spontaneous breathing preterm lamb model.
\end{abstract}

METHODS: Preterm lambs $(n=12)$ of $133-134$ d gestational age were randomized to the following three groups: (i) continuous positive airway pressure (CPAP) only, (ii) CPAP + LISA, and (iii) intubation and mechanical ventilation with surfactant administration. Surfactant was labeled with samarium oxide. During the next $180 \mathrm{~min}$, blood gas analyses were performed. Postmortem, lungs were removed and surfactant distribution was assessed, and pressure-volume curves were performed.

RESULTS: $\mathrm{PaO}_{2}$ in the LISA-treated lambs was significantly higher than in the lambs that exclusively received CPAP. Moreover, $\mathrm{PaO}_{2}$ values were similar between the LISA-treated and the intubated lambs. Overall, surfactant deposition was less in the LISA lambs, with significantly less surfactant distributed to the right upper lobe. Lung compliance was better in the intubated lambs compared with the LISA-treated lambs, although this did not reach significance.

CONCLUSION: LISA improved oxygenation, similar to conventional surfactant application techniques, despite lower surfactant deposition and lung compliance.

$\mathrm{n}$ the past decades, preterm infants have been treated with surfactant bolus replacement therapy for respiratory distress syndrome, which usually requires intubation and mechanical ventilation. Surfactant treatment in ventilated infants leads to a relative reduction in bronchopulmonary dysplasia, pneumothorax, and mortality $(1,2)$. Randomized trials indicated that early surfactant therapy compared to delayed rescue surfactant treatment resulted in better respiratory and neurologic outcomes in preterm infants (3).
Early surfactant treatment has therefore been combined with intubation and mechanical ventilation. Intubation of the trachea may lead to laryngeal or tracheal injury and requires premedication, which can delay the time to extubation. In addition, mechanical ventilation can damage the vulnerable preterm lungs. It has been hypothesized that avoidance of mechanical ventilation might lead to less bronchopulmonary dysplasia $(4,5)$, as infants that failed initial continuous positive airway pressure (CPAP) therapy and needed subsequent mechanical ventilation showed higher bronchopulmonary dysplasia rates (6).

In order to avoid the negative effects of mechanical ventilation, a new technique was recently introduced to administer surfactant endotracheally to spontaneously breathing preterm infants by placing a small feeding or arterial catheter through the vocal cords $(7,8)$. This technique proved to be feasible in clinical practice with first attempt success rate above $80 \%$ (8-10). This technique was referred to as minimally invasive surfactant therapy (8) or less-invasive surfactant administration (LISA) (9). In a multicenter randomized controlled trial, LISA led to a reduction in the need for mechanical ventilation (11). Moreover, a single-center trial showed a reduction in bronchopulmonary dysplasia rates (10). We will further refer to this technique in this study as LISA.

The effects of classical surfactant bolus therapy on oxygenation, gas exchange, and pulmonary surfactant distribution have been studied extensively in experimental setups (12-14). However, this new less-invasive technique has not been studied in animal models. As lung development in fetal and preterm sheep has the same course as in humans, we used the preterm sheep model to study the effects of LISA on oxygenation, gas exchange, pulmonary surfactant distribution, and lung mechanics.

\section{RESULTS}

\section{Basic Characteristics and Oxygenation}

All lambs survived the duration of the experiment (180 min). Heart rate and blood pressure remained within normal values. Basic characteristics are shown in Table 1. 
The intubated lambs showed a swift increase in $\mathrm{PaO}_{2}$ values shortly after intubation and surfactant administration (Figure 1). A similar but slower increase in $\mathrm{PaO}_{2}$ was observed in lambs that received LISA (Figure 1). Only at $t=30 \mathrm{~min}$, $\mathrm{PaO}_{2}$ values were significantly higher in the intubated lambs compared with the lambs that received LISA $(437.0 \pm 60.8$ vs. $197.5 \pm 173.8 \mathrm{mmHg}$ ). When analyzing the spontaneously breathing lambs, $\mathrm{PaO}_{2}$ was significantly higher in the lambs that received LISA than in the lambs that only received CPAP at $t=150$ and $t=180 \mathrm{~min}(355.0 \pm 114.7$ vs. $122.7 \pm 46.3 \mathrm{mmHg}$ and $322.7 \pm 91.0$ vs. $108.0 \pm 52.9 \mathrm{mmHg}$, respectively).

In addition, the oxygenation index $\left(\mathrm{OI}=\mathrm{FiO}_{2} \times\right.$ mean airway pressure $\left(\mathrm{PaO}_{2}\right.$ ) was calculated. Over all time points, the OI was similar between the intubated lambs and the lambs that received LISA $(5.0 \pm 2.1$ vs. $5.1 \pm 4.2)$ (Figure 2). At $t=10, t=$ 30 , and $t=180 \mathrm{~min}$, OI was significantly higher in the lambs that received exclusively CPAP compared with the lambs that received surfactant.

\section{Gas Exchange}

When studying mean $\mathrm{PaCO}_{2}$ over all time points, we found no significant differences in $\mathrm{PaCO}_{2}$ between the groups $(P=0.16$; data not shown).

Table 1. Mean birth weight (g) and umbilical $\mathrm{pH}$ at birth for three different intervention groups

\begin{tabular}{lcccc}
\hline & CPAP & LISA & $\begin{array}{l}\text { Intubation }+ \\
\text { surfactant }\end{array}$ & P value \\
\hline$n$ & 3 & 4 & 5 & \\
Birth weight (g) & $1,556 \pm 338$ & $1,920 \pm 158$ & $1,892 \pm 1,062$ & 0.51 \\
Umbilical pH & $7.30 \pm 0.07$ & $7.22 \pm 0.08$ & $7.17 \pm 0.13$ & 0.17
\end{tabular}

Results are represented as mean with SD.

CPAP, continuous positive airway pressure; LISA, less-invasive surfactant administration.

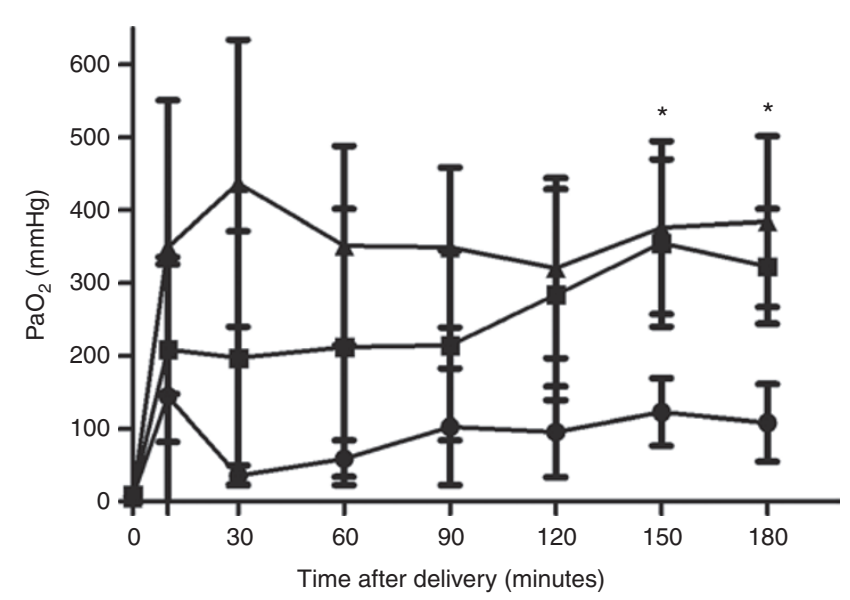

Figure 1. Mean $\mathrm{PaO}_{2}(\mathrm{mmHg})$ in time (minutes) for three different intervention groups. All values are represented as mean with SD. CPAP (filled circles), LISA (filled squares), intubation and surfactant (filled triangles). * Significant difference in $\mathrm{PaO}_{2}$ between the CPAP and LISA group ( $P$ $<0.05)$. CPAP, continuous positive airway pressure; LISA, less-invasive surfactant administration.

\section{Surfactant Deposition}

Total surfactant deposition in the lungs was higher in the lambs that were intubated than in the lambs that received LISA treatment. The amount of the surfactant found in the lungs of the lambs that received LISA was only $17.4 \pm 0.8 \%$ compared to the amount of surfactant found in the lungs of the lambs that were intubated $(P<0.05)$.

Furthermore, exogenous surfactant distribution within each lung lobe was assessed. Surfactant distribution was similar for both the LISA-treated lambs and intubated lambs except for the right upper lobe. In this lobe, less exogenous surfactant was distributed in the LISA-treated lambs compared with the intubated and mechanically ventilated lambs $(0.35 \pm 0.14$ vs. $1.16 \pm 0.87 ; P<0.05$ ) (Figure 3).

Also, the amount of surfactant in the stomach of LISAtreated lambs was assessed. The amount of labeled surfactant in the stomach was invariably low $(1.1 \pm 1.9 \mathrm{mg})$.

\section{Lung Compliance}

Lung compliance was assessed in the lambs that received surfactant with pressure-volume curves. Lung gas volumes were lower in the lambs that received LISA compared with the intubated lambs, although this did not reach significant difference (Figure 4) $(P>0.23)$.

\section{DISCUSSION}

The techniques of LISA to spontaneous breathing infants were introduced into the clinical practice without experimental evaluation $(8,9,11)$. To our knowledge, this is the first study to investigate oxygenation, gas exchange, pulmonary surfactant distribution, and lung compliance in preterm lambs that either exclusively received CPAP, received LISA or were intubated and mechanically ventilated with surfactant administration.

Our findings show that $\mathrm{PaO}_{2}$ values in spontaneously breathing lambs that received LISA were significantly higher than in

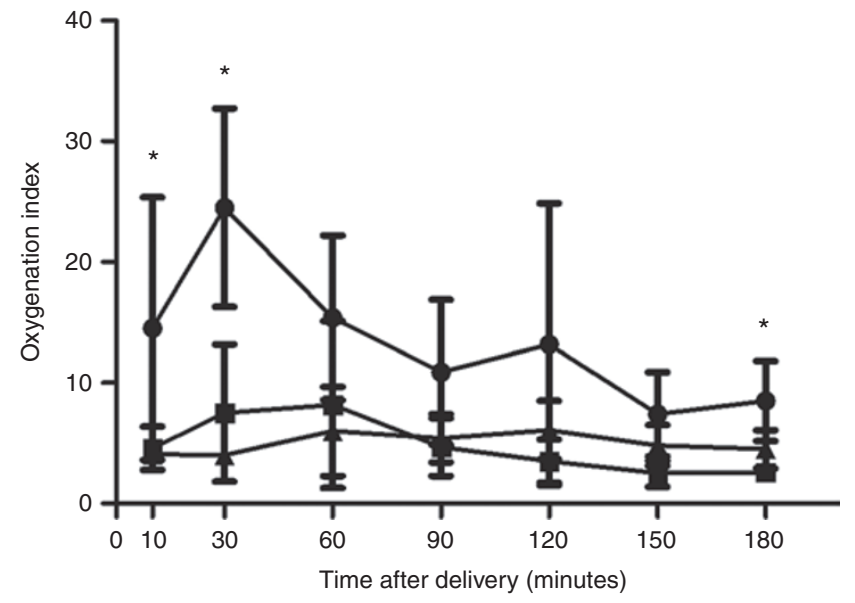

Figure 2. Oxygenation index (OI) for the three different intervention groups. All values are represented as mean with SD. CPAP (filled circles), LISA (filled squares), intubation and surfactant (filled triangles). * Significant difference between CPAP group and LISA and intubated group respectively $(P<0.05)$. CPAP, continuous positive airway pressure; LISA, less-invasive surfactant administration. 


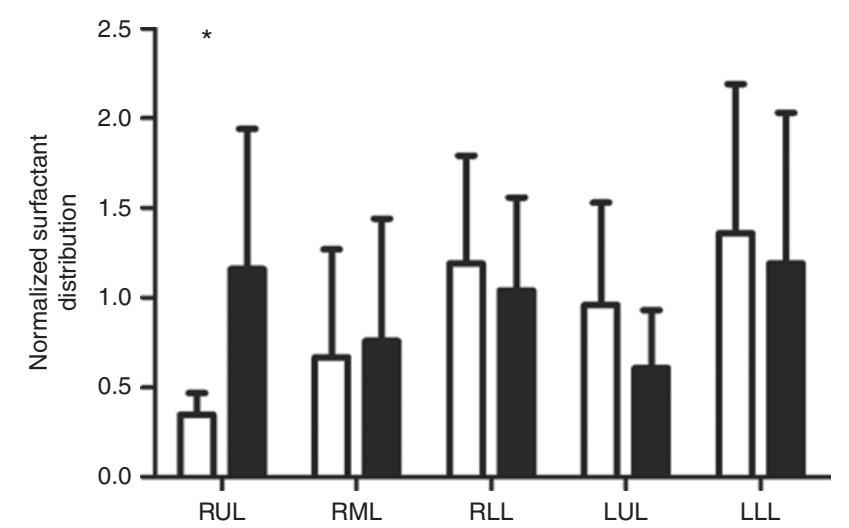

Figure 3. Lobar distribution of exogenous surfactant after less-invasive surfactant administration (LISA) and intubation, surfactant administration, and ventilation. All values are normalized values of surfactant and expressed as means and SD. LISA: white columns; intubation and surfactant: black columns. ${ }^{*} P<0.05$. LLL, left lower lobe; $L U L$, left upper lobe; $\mathrm{RLL}$, right lower lobe; RML, right middle lobe; RUL, right upper lobe.

lambs that received only CPAP and were not significantly different from $\mathrm{PaO}_{2}$ values in intubated and mechanically ventilated lambs. This indicates that surfactant administration by LISA improves oxygenation. Moreover, oxygenation in lambs receiving LISA is similar to the oxygenation in lambs that were intubated and mechanically ventilated and received surfactant.

However, $\mathrm{PaO}_{2}$ values increased faster in the intubated and mechanically ventilated lambs compared with the lambs that underwent LISA. The swift rise in $\mathrm{PaO}_{2}$ values in mechanically ventilated lambs might have been caused by the higher mean airway pressure provided by mechanical ventilation compared to CPAP $\left(19.5 \pm 1.6\right.$ vs. $\left.8.0 \pm 0.0 \mathrm{cmH}_{2} \mathrm{O} ; P<0.01\right)$. The higher mean airway pressure of intubated lambs induces immediate recruitment of lung alveoli and an increase of functional residual capacity, which leads to a better oxygenation. Indeed, the OI, which inherently corrects for mean airway pressure, displayed no differences between the intubated lambs and the lambs that received LISA.

It was striking that the surfactant deposition was significantly lower in the lambs that received LISA compared with the intubated lambs. The exact cause of the lower surfactant deposition with the LISA approach is yet unknown to us. It might have been caused by reflux of surfactant, which was observed in some lambs that received LISA. Reflux of surfactant was observed in the clinical studies as well and may be dependent of the time that is taken for the surfactant administration. As we administered the surfactant rather expeditious (approximately within $1 \mathrm{~min}$ ), this may have led to a reflux of surfactant. However, only low amounts of surfactant were recovered from the stomachs of the lambs that received LISA.

The lower deposition of surfactant may be explained by the fact that when surfactant is administered with LISA, a significant part of the surfactant adheres to the gastric tube, the large airways, and-in case of reflux of surfactant-the esophagus.

Evidence for this explanation is provided by Luca et al. (15). Luca et al. showed that the administration of surfactant through a feeding tube is inferior to the administration

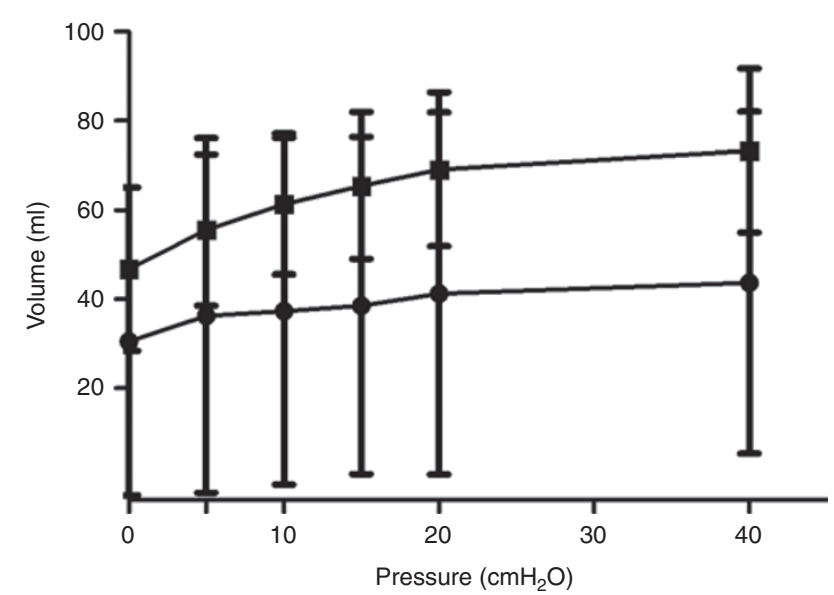

Figure 4. Pressure-volume curve of lambs that received less-invasive surfactant administration (LISA; rounds) and lambs that were intubated and received surfactant (blocks). Results are represented as means and SD. Intubation and surfactant (filled squares) and LISA (filled circles).

through an endotracheal tube because $11 \%$ of the administered surfactant adhered to the feeding tube (15). However, this only partially explains the lower surfactant deposition in the lambs that underwent LISA procedure. Future studies may also focus on the assessment of surfactant deposition in the larger airways and esophagus.

These findings may be used for future adjustments of the dosing of surfactant in LISA-treated infants. Indeed, this concept is supported by a clinical study of Dargaville et al. (8) where none of the infants that received surfactant at a higher dose $(200 \mathrm{mg} / \mathrm{kg}$ ) failed subsequent CPAP therapy. It is a surprising result that, despite lower deposition of surfactant in the lungs, oxygenation was similar between the lambs that received LISA and the intubated lambs. This may be caused by the fact that in spontaneous breathing animals, exogenous surfactant has a higher tissue association and leads to a higher dynamic lung compliance compared to the ventilated animals, as was shown in a rabbit model by Bohlin et al. (16).

The distribution of endotracheally administered surfactant in mechanically ventilated animals has been studied by numerous groups (17-19). The different lung lobes develop in a different sequential pattern with the most advanced developed parts of the lungs being the right and left upper lobes $(20,21)$. We were therefore interested in the comparison of the distribution between the lobes in spontaneously breathing vs. intubated and mechanically ventilated animals. Compared to the intubated lambs, less surfactant was allocated to the right upper lobe of the LISA-treated lambs. As, by the use of LISA, less surfactant is allocated in proportion to the more mature right upper lobe, pulmonary distribution of exogenous surfactant may take place in a more physiologic fashion. This may explain why, despite lower surfactant deposition, the effect on oxygenation and gas exchange of both surfactant application techniques was similar.

Furthermore, in the ventilated lambs that received surfactant, the lungs showed higher compliance than the lungs of the lambs treated with LISA. However, this did not reach 
statistical significance. This might have been caused by higher mean airway pressure or the higher levels of deposited exogenous surfactant in intubated and ventilated lambs. However, the higher compliance in intubated lambs did not lead to better oxygenation. In our opinion, the most clinically relevant endpoint is oxygenation, since preterm infants who fail CPAP therapy show worse oxygenation during the first hours after birth $(6,22)$.

There are several limitations to this study. At first, as stated above, the number of investigated animals was low. A second limitation of the study is the applicability of the experience in preterm lambs to the situation in preterm babies on account of anatomical limitations. The trachea in lambs is longer than in preterm babies resulting in more pressure loss. After birth, we supported the lambs with nasal intermittent positive pressure ventilation, as CPAP only was not sufficient. Therefore, we used a standardized nasal intermittent positive pressure ventilation approach as resuscitation/stabilization approach. This approach is not in accordance with the standard clinical scenario, where stabilization usually occurs with CPAP only.

In conclusion, we demonstrated in preterm lambs that LISA was feasible and improved oxygenation in comparison to standard CPAP support after birth. When comparing the intubated and mechanically ventilated lambs receiving standard surfactant administration to the spontaneous breathing lambs receiving LISA, there were no differences in oxygenation and gas exchange, despite lower pulmonary surfactant deposition and lung compliance in LISA-treated lambs.

\section{METHODS}

\section{Experimental Protocol}

The experimental protocol and design of the study were in line with the institutional guidelines for animal experiments and were approved by the Institutional Animal Ethics Research Committee of the Maastricht University. Singleton lambs of time-mated Texel ewes were delivered by Caesarean section under spinal anesthesia and midazolam sedation at 133-134 d gestational age (term $=147 \mathrm{~d}$ gestational age). Directly after birth, the preterm lambs were stabilized with nasal intermittent positive pressure ventilation using a Babylog 8000 ventilator (Dräger, Lübeck, Germany). We followed a standardized protocol, which was described earlier (PIP $30 \mathrm{cmH}_{2} \mathrm{O}$, positive end-expiratory pressure (PEEP) $8 \mathrm{cmH}_{2} \mathrm{O}$, respiratory rate $60 / \mathrm{min}$, fraction of inspired oxygen $\left(\mathrm{FiO}_{2}\right)=1.0(23)$. All lambs received umbilical arterial and venous catheters. All lambs received parenteral feeding during the experiment. Rectal temperature, heart rate, and blood pressure were continuously monitored.

The lambs were randomly assigned to three treatment groups after 5 min of nasal intermittent positive pressure ventilation. In all groups, $\mathrm{FiO}_{2}$ remained 1.0 during the entire experiment:

1. CPAP group: $\mathrm{CPAP}$ with PEEP of $8 \mathrm{cmH}_{2} \mathrm{O}$ without surfactant administration $(n=3)$.

2. LISA group: CPAP with PEEP $8 \mathrm{cmH}_{2} \mathrm{O}$ with endotracheal lessinvasive administration of surfactant (Poractant $\alpha, 200 \mathrm{mg} / \mathrm{kg}$ ) through a gastric catheter $(n=4)$. The surfactant was a gift from Chiesi Pharmaceuticals (Parma, Italy). The procedure of LISA was the following. A 6 French gauge feeding tube (Vygon, Swindon, UK) was inserted $3 \mathrm{~cm}$ beyond the vocal cords with direct laryngoscopy. After $30 \mathrm{~s}$ without bradycardia, surfactant instillation was started. The surfactant was administered with direct observation of the position of the catheter or potential reflux of surfactant. When reflux or bradycardia was observed, the procedure was interrupted. Surfactant was administered within $1 \mathrm{~min}$. Hereafter, the gastric tube was immediately removed. CPAP support was not disrupted during the whole procedure.

3. Intubation and surfactant group: intubation and mechanical ventilation with endotracheal administration of surfactant through the endotracheal tube (Vygon, $3.5 \mathrm{~mm}$ cuffed; Poractant $\alpha, 200 \mathrm{mg} / \mathrm{kg})(n=5)$. Initial ventilator settings for mechanical ventilation were: peak inspiratory pressure of 30 $\mathrm{cmH}_{2} 0$, PEEP of $8 \mathrm{cmH}_{2} \mathrm{O}$, inspiratory time of $0.4 \mathrm{~s}$, and frequency of $60 / \mathrm{min}$. Peak inspiratory pressure was adjusted in order to achieve tidal volumes $5-10 \mathrm{ml} / \mathrm{kg}$ and a target $\mathrm{pCO}_{2}$ of $50 \mathrm{mmHg}$. The ventilated lambs were sedated with midazolam $(0.3 \mathrm{mg} / \mathrm{kg} / \mathrm{h})$ and fentanyl $(5 \mu \mathrm{g} / \mathrm{kg} / \mathrm{h})$.

The surfactant was labeled with samarium oxide $\left(\mathrm{Sm}_{2} \mathrm{O}_{3}\right)$, which is a nonradioactive tracer. The batches contained $1.0-\mu \mathrm{g} \mathrm{Sm}_{2} \mathrm{O}_{3} / \mathrm{mg}$ surfactant.

In the next $180 \mathrm{~min}$, blood gas analyses through the umbilical arterial catheter were performed 10 and $30 \mathrm{~min}$ after birth and subsequently at 30-min intervals (Abott i-STAT 1 Blood Gas Analyzer; Abbott Laboratories, Chicago, IL). Hereafter, the lambs were euthanized by pentobarbital infusion $(200 \mathrm{mg} / \mathrm{kg})$.

\section{Postmortem Analysis}

After euthanasia, the lambs were disconnected from the ventilator. From the lambs that received surfactant, the thorax was opened by a midline incision as previously described (24). An endotracheal tube was inserted when necessary and connected to a manometer. A deflation limb of a pressure-volume curve was recorded with a maximum pressure of $40 \mathrm{cmH}_{2} \mathrm{O}$. All lung volumes are expressed as milliliters per kilogram body weight.

All five lung lobes were isolated, weighed, and used for the analysis of surfactant distribution by quantification of $\mathrm{Sm}_{2} \mathrm{O}_{3}$. For that means, the frozen lung tissues were further processed (freeze dried, ashed, and digested with a combination of hydrochloric acid, nitric acid, and water) and afterward analyzed by inductively coupled plasma mass spectrometry with a XSERIES II ICP MS (Thermo Fisher Scientific, Breda, The Netherlands) (23). Stomach contents were analyzed for the presence of exogenous surfactant as well.

Pulmonary surfactant concentration was defined as $\mathrm{Sm}_{2} \mathrm{O}_{3}$ weight per lung lobe weight and was measured in each lung lobe. Pulmonary surfactant deposition in each lung lobe was expressed as normalized value as was earlier performed by Dijk et al. (17). The normalized value was calculated by dividing the weight of $\mathrm{Sm}_{2} \mathrm{O}_{3}$ per gram of tissue for each lung piece by the average value of $\mathrm{Sm}_{2}^{2} \mathrm{O}_{3}$ per gram of tissue of all the lung pieces for each lamb (mean $\mathrm{Sm}_{2}^{2} \mathrm{O}_{3}$ concentration).

\section{Statistical Analysis}

Statistical analysis was performed with IBM SPSS for Windows version 20.0 (IBM, Armonk, NY). Results were analyzed with one-way ANOVA, with post hoc testing for differences between each group. Results are represented with mean and SD.

\section{STATEMENT OF FINANCIAL SUPPORT}

The surfactant used in this study was a gift from Chiesi Pharmaceuticals. The authors have no affiliation, no financial agreement, or other involvement with any company.

\section{REFERENCES}

1. Halliday HL. Surfactants: past, present and future. J Perinatol 2008;28:Suppl 1:S47-56.

2. Pfister RH, Soll RF. Initial respiratory support of preterm infants: the role of CPAP, the INSURE method, and noninvasive ventilation. Clin Perinatol 2012;39:459-81.

3. Halliday HL. History of surfactant from 1980. Biol Neonate 2005;87:31722.

4. Finer N. To intubate or not-that is the question: continuous positive airway pressure versus surfactant and extremely low birthweight infants. Arch Dis Child Fetal Neonatal Ed 2006;91:F392-4.

5. Jobe AH. Transition/adaptation in the delivery room and less RDS: "Don't just do something, stand there!". J Pediatr 2005;147:284-6. 


\section{Articles | Niemarkt et al.}

6. Dargaville PA, Aiyappan A, De Paoli AG, et al. Continuous positive airway pressure failure in preterm infants: incidence, predictors and consequences. Neonatology 2013;104:8-14.

7. Kribs A, Vierzig A, Hünseler C, et al. Early surfactant in spontaneously breathing with nCPAP in ELBW infants-a single centre four year experience. Acta Paediatr 2008;97:293-8.

8. Dargaville PA, Aiyappan A, De Paoli AG, et al. Minimally-invasive surfactant therapy in preterm infants on continuous positive airway pressure. Arch Dis Child Fetal Neonatal Ed 2013;98:F122-6.

9. Klebermass-Schrehof K, Wald M, Schwindt J, et al. Less invasive surfactant administration in extremely preterm infants: impact on mortality and morbidity. Neonatology 2013;103:252-8.

10. Kanmaz HG, Erdeve O, Canpolat FE, Mutlu B, Dilmen U. Surfactant administration via thin catheter during spontaneous breathing: randomized controlled trial. Pediatrics 2013;131:e502-9.

11. Göpel W, Kribs A, Ziegler A, et al.; German Neonatal Network. Avoidance of mechanical ventilation by surfactant treatment of spontaneously breathing preterm infants (AMV): an open-label, randomised, controlled trial. Lancet 2011;378:1627-34.

12. Fujiwara T, Maeta H, Chida S, Morita T, Watabe Y, Abe T. Artificial surfactant therapy in hyaline-membrane disease. Lancet 1980;1:55-9.

13. Rider ED, Jobe AH, Ikegami M, Sun B. Different ventilation strategies alter surfactant responses in preterm rabbits. J Appl Physiol (1985) 1992;73:2089-96.

14. van der Bleek J, Plötz FB, van Overbeek FM, et al. Distribution of exogenous surfactant in rabbits with severe respiratory failure: the effect of volume. Pediatr Res 1993;34:154-8.

15. Luca DD, Minucci A, Gentile L, Capoluongo ED. Surfactant inadvertent loss using feeding catheters or endotracheal tubes. Am J Perinatol 2014;31:209-12.
16. Bohlin K, Bouhafs RK, Jarstrand C, Curstedt T, Blennow M, Robertson B. Spontaneous breathing or mechanical ventilation alters lung compliance and tissue association of exogenous surfactant in preterm newborn rabbits. Pediatr Res 2005;57(5 Pt 1):624-30.

17. Dijk PH, Heikamp A, Oetomo SB. A comparison of the hemodynamic and respiratory effects of surfactant instillation during interrupted ventilation versus noninterrupted ventilation in rabbits with severe respiratory failure. Pediatr Res 1999;45:235-40.

18. Segerer H, van Gelder W, Angenent FW, et al. Pulmonary distribution and efficacy of exogenous surfactant in lung-lavaged rabbits are influenced by the instillation technique. Pediatr Res 1993;34:490-4.

19. Ueda T, Ikegami M, Rider ED, Jobe AH. Distribution of surfactant and ventilation in surfactant-treated preterm lambs. J Appl Physiol (1985) 1994;76:45-55.

20. Brumley GW, Crenshaw C. Fetal lamb lung phosphatidylcholine: response to asphyxia and recovery. J Pediatr 1980;97:631-4.

21. Pringle KC. Human fetal lung development and related animal models. Clin Obstet Gynecol 1986;29:502-13.

22. Fuchs H, Lindner W, Leiprecht A, Mendler MR, Hummler HD. Predictors of early nasal CPAP failure and effects of various intubation criteria on the rate of mechanical ventilation in preterm infants of $<29$ weeks gestational age. Arch Dis Child Fetal Neonatal Ed 2011;96:F343-7.

23. Rahmel DK, Pohlmann G, Iwatschenko P, et al. The non-intubated, spontaneously breathing, continuous positive airway pressure (CPAP) ventilated pre-term lamb: a unique animal model. Reprod Toxicol 2012;34: 204-15.

24. Seehase M, Collins JJ, Kuypers E, et al. New surfactant with SP-B and C analogs gives survival benefit after inactivation in preterm lambs. PLoS One 2012;7:e47631. 